\title{
LA CIRCULACIÓN DE LA ESCUELA \\ NUEVA EN BRASIL (1933-I958): ENTRE PRESCRIPCIONES E INNOVACIONES
}

\section{The circulation of the "New School» in Brazil (1933-1958): between prescriptions and innovations}

\author{
Vera Teresa Valdemarin \\ Universidade Estadual Paulista (UNESP). Araraquara (Brasil) \\ Correo-e: veravaldemarin@gmail.com \\ Manuela Priscila De Lima Bueno \\ Universidade Estadual Paulista (UNESP). Araraquara (Brasil)
}

Recepción: io del abril de 20I6. Envío a informantes: I8 de abril de 2016 Aceptación definitiva: I de agosto de 2016

RESUMEN: El presente artículo enfoca la circulación de las concepciones vinculadas al ideario de la Escuela Nueva en Brasil entre los años 1930 y (1958?), desde las prescripciones destinadas a introducir nuevas prácticas pedagógicas en las instituciones formadoras y, por medio de ellas, atender las demandas sociales y educacionales del país. Tomando como fuentes documentales la legislación educacional, los programas prescritos para la formación de profesores e informes producidos por comisarios de la enseñanza, se procura reunir orientaciones producidas en diferentes instancias de poder para captar la combinación entre léxico renovador, limitaciones del sistema educacional y prácticas que se pretendía diseminar y que, al final, produjeron tensión entre concepciones y experiencias prácticas, caracterizando una innovación marcada por la apropiación selectiva de las pretensiones de la renovación pedagógica.

Palabras Clave: educación brasileña; Escuela Nueva; formación de profesores; prácticas pedagógicas; instituciones formadoras.

AвSTRACT: This article focuses on the circulation of ideas linked to the ideology of the «New School» in Brazil between 1930 and 1958 , from the prescriptions aimed at introducing new pedagogical practices in training institutions and, through them, meet the social and educational demands of the country. By having the educational legislation, the prescribed programs for teacher training, and reports produced by 
delegates of education as documentary sources, this article seeks to gather guidelines produced in different instances of power to capture the combination of a refreshing lexicon, limitations of the educational system and practices intended to be spread, which, in the end, caused tension between concepts and practical experiences, featuring an innovation marked by selective appropriation of the pedagogical renewal pretensions.

KeY words: Brazilian education; New Schools; teachers training; pedagogical practices; institutions.

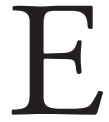
L PROCESO DE CIRCULACIÓN DE LAS IDEAS PEDAGÓGICAS es muy complejo y su movimiento no lineal entrelaza actores, instituciones y un conjunto de acciones que se ramifican en cambios legales, iniciativas editoriales y ocupación de posiciones en el sistema educacional. La comprensión de esa dinámica ha movilizado el interés de investigadores brasileños, de manera especial, cuando se trata de las concepciones tributarias de la Escuela Nueva o Educación Progresiva, que se convirtió en hegemónica en los años de 1930.

Diferentes estudios sobre esa temática marcaron el itinerario de las realizaciones más significativas y los liderazgos más expresivos en la difusión de las ideas y de los valores alineados con las transformaciones entonces pretendidas en el

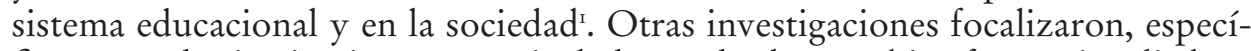
ficamente, las instituciones a partir de las cuales los cambios fueron irradiados ${ }^{2}$, las iniciativas editoriales destinadas a la difusión de las concepciones consideradas innovadoras ${ }^{3}$ y los autores de los textos se convirtieron en emblemáticos en la

Se deben mencionar, principalmente, los análisis de Nagle, J.: Educação e Sociedade na Primeira República, São Paulo, EPU/Rio de Janeiro, Fundação Nacional de Material Escolar, 1974; CARvalho, M. M. C.: A escola e a república, São Paulo, Brasiliense, 1989; Carvalho, M. M. C.: O molde nacional e a forma cívica: higiene, moral e trabalho no projeto da Associação Brasileira de Educação (1924-I93I), Bragança Paulista: EDUSF, I998; NunEs, C.: «História da educação brasileira: novas abordagens de velhos objetos», Teoria E Educação, n. 6 (1992), pp. I5I-182; SAviani, D.: História das ideias pedagógicas no Brasil, Campinas, Autores Associados, 2007.

VIDAL, D. G.: O exercício disciplinado do olhar: livros, leituras e práticas de formação docente no Instituto de Educação do Distrito Federal (1932-1937), Bragança Paulista, EUSF, 20oI; Lopes, S. C.: Oficina de mestres. História, memória e silêncio sobre a Escola de professores do Instituto de Educação do Rio de Janeiro (1932-1939), Rio de Janeiro, DP\&A/FAPERJ, 2006; PINTO, K. P.: Por uma nova cultura pedagógica: prática de ensino como eixo da formação de professores primários do Instituto de Educação do Rio de Janeiro (1932-1937), Tese (Doutorado em Educación), São Paulo, Pontifícia Universidade Católica, 2006; Arce, A. y Nery, A. C. B.: Ideias pedagógicas em movimento: produção de saberes na Escola Normal Secundária de São Carlos, São Carlos, Edufscar, 201 I.

3 Bastos, M. H. C.: A revista do Ensino-Rs: o novo e o nacional em revista, Pelotas, Seiva, 2005; Carvalho, M. M. C. y Toledo, M. R. A.: «A Biblioteca da Educação de Lourenço Filho: uma coleção a serviço de um projeto de inovação pedagógica», Quaestio, 2 (8) (2006), pp. 47-62; Toledo, M. R. A.: Coleção Atualidades pedagógicas: do projeto político ao projeto editorial (1931-198I), Tese (Doutorado em Educação), São Paulo, Pontifícia Universidade Católica de São Paulo, 2oor; Nery, A. C. B.: A sociedade de educação de São Paulo: embates no campo educacional paulista (1922-1931), São Paulo, edunesp, 2009; Valdemarin, V. T.: História dos métodos e materiais de ensino: a escola nova e seus modos de uso, São Paulo, Cortez, 2oio; MAte, C. H.: Tempos modernos na escola. Os anos 30 e a racionalização da educação brasileira, Bauru, EDUsc/Brasília, INEP, 2002. 
LA CIRCULACIÓN DE LA ESCUELA NUEVA EN BRASIL (I933-I958):

ENTRE PRESCRIPCIONES E INNOVACIONES

VERA TERESA VALDEMARIN Y MANUELA PRISCILA DE LIMA BUENO

propuesta de nuevos rumbos educacionales ${ }^{4}$. El conjunto de pesquisas referenciadas indica que, bajo la adhesión a los propósitos renovadores, los sentidos de la innovación fueron construidos en diferentes contextos de recepción y de interpretación.

Insertándose en esa temática, el presente artículo focaliza la circulación de las concepciones vinculadas al ideario de la Escuela Nueva a partir de las prescripciones destinadas a introducir nuevas prácticas pedagógicas en las instituciones formadoras y, a través de ellas, atender lo que se entendía que eran las demandas sociales y educacionales del país. Se trata aquí de analizar el arraigamiento y los significados adquiridos por la renovación entonces pretendida, a fin de comprender la correlación entre formas de pensar y modos de hacers. Teniendo en cuenta que las innovaciones ocurren en una red de relaciones por divergencias y consensos, se pretende focalizar lo que Raymond Williams ${ }^{6}$ denominó continuidades prácticas presentes en los procesos culturales, esto es, lugares, instituciones y lenguajes que son directamente probados y revelan la existencia de una apropiación selectiva de las pretensiones de la renovación pedagógica.

Ese proceso es discutido aquí en la perspectiva de la formación de profesores -factor estratégico para la elevación de la cultura profesional, propagación del espíritu científico y vinculación de la escuela en el entorno social- ejemplificada en la iniciativas del estado de São Paulo, entre los años de 1930 y 1958. Tomando como fuentes documentales la legislación educacional, los programas prescritos para la formación de profesores e informes producidos por delegados de enseñanza, se busca reunir orientaciones producidas en diferentes foros de poder para captar la combinación entre el léxico renovador, las limitaciones del sistema educacional y las prácticas que se pretendía diseminar y que, al fin, produjeron «una innovación infiltrada en los términos de una tradición»?

\section{Estrategias de la renovación}

En la historiografía de la educación brasileña, la expresión Escuela Nueva ha designado el movimiento educacional que emergió en los años 1920 y 1930, fue el motivo de una intensa disputa entre educadores católicos y liberales y pretendió romper con prácticas y métodos pedagógicos tenidos como tradicionales, alineándose al desarrollo urbano industrial preconizado para el país. Entre los principales rasgos de la reconstrucción nacional por la reconstrucción de la educación

4 Monarcha, C. R. S (org.): Lourenço Filho. Outros aspectos, mesma obra, Campinas, Mercado de Letras, 1997; Cunha, M. V.: John Dewey - a utopia democrática, Rio de Janeiro, DP\&A, 20oI; Cunha, M. V.: «O manifesto dos pioneiros de 1932 e a cultura universitária brasileira: razão e paixão», Revista Brasileira de História da Educação, n. 17 (2008), pp. I24-I4I; Xavier, M. C. (org.): Manifesto dos pioneiros da educação: um legado educacional em debate, Rio de Janeiro, FGV, 2004.

Certeau, M.: A invenção do cotidiano: artes de fazer, Petrópolis, Vozes, 2005.

Williams, R.: Marxismo e literatura, Rio de Janeiro, Zahar, 1979.

Certeau: op. cit., p. 50. 
(uno de los eslóganes del movimiento) se puede apuntar la pretensión de redireccionar el papel del Estado en la educación, expandir la escuela pública para garantizar a todos el derecho a la educación, racionalizar el sistema articulando modalidades y grados de instrucción y modificar las relaciones entre aprendizaje y enseñanza ${ }^{8}$.

Las pretensiones y la amplitud de las reformas que serán desencadenadas fueron expresadas en el Manifesto dos Pioneiros da Educação Nova ${ }^{9}$, documento que revela el

intento en producir un efecto de verdad: compartir la educación brasileña, de extremo a extremo entre el nuevo y el viejo, como todas las demás dimensiones de la vida nacional, teniendo como base el diagnóstico de que el nuevo emergía en su plenitud en el Brasil porque el viejo resistía y se negaba a la muerte, aunque ya a ella estuviera condenado en otros países $[\ldots]^{\text {to }}$.

El movimiento no se restringía a la discusión de los principios o de las finalidades educacionales y sociales, como señala Marta Carvalho:

En la lucha por el control del aparato escolar, la confrontación doctrinal en el entorno de la Pedagogía tuvo papel central, pues en él estaban siendo normativizadas las prácticas escolares. Es en esa lucha que, con diferentes estrategias, los propagandistas de la denominada pedagogía de la escuela nueva reivindicaban el monopolio de lo nuevo y de lo moderno, produciendo, peyorativamente, los saberes pedagógicos concurrentes como pedagogía tradicional" ${ }^{\mathrm{II}}$.

Con tantas pretensiones en juego, las interpretaciones sobre el movimiento son, necesariamente, multifacéticas y, dependiendo de las fuentes documentales utilizadas, diferentes aspectos pasan a integrar la construcción de sentidos entrelazados $^{12}$. En el análisis, la delimitación temporal está enmarcada por dos

8 Esas iniciativas brasileñas no fueron aisladas. Franco Cambi afirma que innumerables proposiciones desarrolladas, principalmente en Europa y en los Estados Unidos, «tuvieron inmediatamente amplia resonancia en el mundo educativo, propiciaron una serie de pesquisas en el campo de la instrucción, destinadas a transformar profundamente la escuela no solo en su aspecto organizacional e institucional, sino también, y quizás sobre todo, en el aspecto relacionado con los ideales formativos y los objetivos culturales». CAmbi, F.: História da pedagogia, São Paulo, Edunesp, 1999, p. 5I4. De la misma forma, se debe señalar que el movimiento no era homogéneo, pero sí constituido por diferentes interpretaciones de los caminos necesarios para la renovación.

9 En 1932, ese documento fue publicado en la prensa diaria, en el periódico Educação y en el libro A reconstrução educacional no Brasil: ao povo e ao governo, ordenado por Fernando Azevedo, cf. CunHa: 2008, op. cit., y ha sido reproducido en muchas obras, dada la connotación que adquirió a lo largo del tiempo.

ro Warde, M. J.: «Legado e legatários: questões sobre o Manifesto dos Pioneiros da educação nova», en Xavier, M. C. (org.): op. cit., p. 229.

" Carvalho, M. M. C.: «Invenções e tradições na história da educação no Brasil: questionando representações sobre o debate pedagógico na década de I930», en SIMÕES, R. H. S. E. y GondRA, J. G.: Invenções, tradições e escritas da História da Educação, Vitória, EDUFEs, 20I2, p. IO.

${ }_{12}$ Para un abordaje nacional del movimiento: Miguel, E. B.; Vidal, D. G. y Araujo, J. C. S. (orgs.): Reformas educacionais: as manifestações da Escola Nova no Brasil, Campinas, Autores Associados, 20II. 
LA CIRCULACIÓN DE LA ESCUELA NUEVA EN BRASIL (I933-I958):

ENTRE PRESCRIPCIONES E INNOVACIONES

VERA TERESA VALDEMARIN Y MANUELA PRISCILA DE LIMA BUENO

reformas educacionales de gran porte ${ }^{\mathrm{I} 3} \mathrm{y}$ las fuentes documentales - determinaciones legales generales y reglamentaciones específicas para nortear cotidianamente el trabajo en las instituciones- ofrecen informaciones sobre el desarrollo de ese proceso, o sea, sobre la acción sistémica de diferentes agentes que interfirieron en la formación y en la institucionalización de las prácticas renovadas.

El dispositivo legal fue el instrumento más utilizado para delimitar los cambios pretendidos. En el estado de São Paulo, en 1933, bajo la gestión de Fernando de Azevedo, fue promulgado el Código de Educación ${ }^{14}$, un largo documento, compuesto por más de 900 artículos, que explicita inequívocamente la intención de alterar el sistema educativo una vez que «exigencias de nuestra situación social y económica imponen la reorganización de nuevas bases, de las instituciones escolares existentes» ${ }^{15}$. El documento prioriza la creación del aparato administrativo, detallando su forma organizacional, y anuncia la renovación pedagógica que ésta posibilitaría. Están allí definidas las finalidades de la educación pública y de cada sector y grado de instrucción específicos (educación preescolar, educación primaria, educación prevocacional, educación técnico-profesional, educación secundaria, educación pedagógica, educación especializada) subordinadas al Departamento de Educación. Para viabilizar el funcionamiento de esta estructura, el estado de São Paulo fue dividido en 2r regiones, para las cuales fueron designados delegados de Enseñanza que, auxiliados por los inspectores escolares, cuidarían tanto de la fiscalización como de la orientación del trabajo realizado en las escuelas por directores y profesores.

La intención de crear un soporte administrativo propicio al desarrollo de las acciones pedagógicas de carácter científico permeaba el Código y se revelaba en la atribución de funciones. Al almacenamiento, por ejemplo, responsable de guardar y comprar muebles y materiales «apropiados al mejor desarrollo de los programas escolares» competía también el «estudio experimental de la renovación del material y mobiliario escolar [...] más adecuados al trabajo en común y a la mayor libertad de acciones de los alumnos» ${ }^{16}$.

Esa directriz adquiría especificidades en los diferentes niveles del sistema educacional. El Servicio de Educación Infantil debería "promover la iniciación moral y social, apoyada en una disciplina de autodirección y autocontrol» [...] «vulgarizar los conocimientos científicos sobre el niño» y «propagar los sistemas de Froe-

${ }_{13}$ La primera se materializa en el Código de Educação promulgado en 1933 y la segunda en la Lei de Diretrizes e Bases, promulgada en 196I (BrasiL: «Lei n. 4.024, a 20 de diciembre de 196r. Lei de Diretrizes e Bases da Educação Nacional», Diário Oficial da República Federativa do Brasil, Brasília, DF, 196I). Además, la primera sea restricta al estado de São Paulo, describe, de forma precursora, la organización administrativa necesaria para que el sistema educacional pudiese funcionar en nuevos modelos; la segunda, resultante de un largo proceso de tramitación, pretendió redefinir la actuación del Estado y de otras esferas en la educación.

${ }_{14}$ São Paulo: Decreto n. 5.884, de 21 de abril de 1933. Institue o Código de Educação do Estado de São Paulo, Imprensa Oficial do Estado de São Paulo, 1933 (Coleção de leis e decretos do Estado de São Paulo).

is Op. cit., Introducción.

16 Op. cit., artículo 23. 
LA CIRCULACIÓN DE LA ESCUELA NUEVA EN BRASIL (I933-I958):

ENTRE PRESCRIPCIONES E INNOVACIONES

VERA TERESA VALDEMARIN Y MANUELA PRISCILA DE LIMA BUENO

bel y Montessori y otros, como punto de partida para estudios e investigaciones que inspiren adaptaciones de métodos y procesos, de acuerdo con las características de nuestro entorno ${ }^{17}$; el Servicio de Clasificación y Promoción de Alumnos debería actuar «en la formación de la consciencia técnica de los profesores» indicando las ventajas del empleo de los tests psicológicos y pedagógicos y divulgando estudios sobre la aplicación de la Psicología al campo educacional ${ }^{18}$ bien cómo gestionar iniciativas experimentales; al Servicio de Programas y Libros Escolares competería formar comisiones para analizar libros y material didáctico, ordenar investigaciones sobre la lectura, incentivar la publicación y la traducción de obras, promover conferencias y «conducir la organización de programas mínimos generales [...] reconstruyéndolos científicamente, a través de procesos objetivos de investigación y de medida, sobre los datos de estudios de los grupos sociales y de la psicología genética ${ }^{19}$ ». El Servicio de Publicidad e Informaciones debería publicar revistas, monografías y sinopsis de los trabajos realizados, para, entre otras acciones, divulgar conocimiento, registrar iniciativas y bibliografía y, reuniendo todas las informaciones, la publicación del Anuario de Enseñanza consolidaría el trabajo del Departamento de Educación presentando los balances realizados por los varios servicios técnicos y por los delegados regionales escolares.

La caracterización del Instituto de Educación de la capital del estado como polo generador y diseminador de nuevos modelos de actuación pedagógica también es estrategia inequívoca en el Código de Educación ${ }^{20}$. El Instituto de Educación Caetano de Campos (nomenclatura adoptada a partir de 1933) reunía Escuela de Profesores, Escuela Secundaria, Escuela Primaria, Guardería, Servicio de Psicología Aplicada y Biblioteca, que hacían de la práctica de enseñanza desarrollada en nuevos modelos el elemento clave para la formación de profesores. Los cursos ofrecidos en el Instituto de Educación deberían convertirse en oportunidad para el desarrollo de la experimentación pedagógica, una vez que en ellos actuaban profesores «rigurosamente elegidos en el cuadro de magisterio primario ${ }^{21}$ y, por esa vía, contribuir a la formación de maestros, inspectores y directores escolares. El profesor de Práctica de Enseñanza de la Escuela de Formación de Profesores era también director de la Escuela Primaria y de la Guardería, que

\footnotetext{
Op. cit., artículo 37 .

Op. cit., artículo 95 .

Op. cit., artículo Io4.
}

20 En esa estrategia ya había sido implementada por Fernando de Azevedo en la reforma educacional de Río de Janeiro, entonces Distrito Federal, 1928, y creó las condiciones para que la formación de profesores fuera elevada al nivel superior de instrucción. Se entrelazaban las experiencias y los resultados en la formación de profesores primarios, la demanda para la formación de profesores secundarios y los proyectos en discusión para la creación de las Universidades. El curso de formación ocupaba una posición singular en el sistema educacional: exigía como requisito previo el curso secundario, diferenciándose de los otros cursos profesionales y su forma de organización se materializó en el Instituto de Educación y se le acercaron a las metas establecidas para la educación superior: la transmisión del conocimiento alineada a la investigación. En 1938, el Instituto de Educación y la formación en él ofrecida volvieron al nivel secundario de instrucción.

${ }^{21}$ SÃo PaUlo: op. cit., artículo 768. 
LA CIRCULACIÓN DE LA ESCUELA NUEVA EN BRASIL (I933-I958):

ENTRE PRESCRIPCIONES E INNOVACIONES

VERA TERESA VALDEMARIN Y MANUELA PRISCILA DE LIMA BUENO

servían como campo para «observación, experimentación y práctica de métodos y procesos de enseñanza» ${ }^{22}$.

Así, se puede decir que, estableciendo significados para el ideario de la Escuela Nueva, el Código de Educación del Estado de São Paulo determinó las líneas generales a ser seguidas en el sistema educacional y, específicamente, en la formación de profesores. Entre éstos estaban la valoración de la investigación para la generación de conocimientos e informaciones, la divulgación de nuevas referencias para la práctica pedagógica y la organicidad del sistema funcionando bajo una misma concepción.

Para pavimentar el trayecto entre las intenciones explicitadas en la ley general y las acciones desarrolladas en las escuelas, diferentes dispositivos impresos fueron producidos y, entre ellos, se destacan los programas para las asignaturas componentes del curso de formación de profesores. Divulgados por el Departamento de Educación, los programas se destinaban a administrar la diseminación de la renovación, indicando el contenido a ser enseñado, la bibliografía, la carga horaria y la organización curricular; construirían, por esa vía, un glosario pedagógico indicativo de las prácticas formativas y de los autores de referencia más adecuados a los futuros profesores. El análisis de los Programas revela, por lo tanto, modos para materializar las intenciones legales, interviniendo directamente en el aula.

En 1936, fueron publicados los Programas dos Cursos Regulares e Extraordinários em funcionamento no Instituto de Educação ${ }^{23}$ de la capital (entonces vinculado a la universidad). En el programa de la asignatura de Sociología Educacional, elaborado por Fernando de Azevedo para el curso de Formación de Profesores, la pesquisa era práctica prioritaria, una vez que el abordaje conceptual de los temas venía acompañado de sugerencias de trabajo para amparar la constitución de grupos de estudios, la realización de investigaciones, pesquisas y monografías sobre la sociedad, sobre las condiciones y los sistemas escolares y sobre las características sociales de los estudiantes.

El programa de Filosofía de Educación listaba los temas centrales de la Escuela Nueva en su vertiente norteamericana, tales como la naturaleza de las instituciones en una civilización en cambio, la relación entre experiencia y pensamientos y entre intereses y asignatura. El Programa de Educación Comparada situaba la renovación ocurrida en diferentes países, insertando Brasil en ese panorama; en el programa de Metodología de la Enseñanza Primaria, entre las cuestiones propias de la didáctica general, estaban presentes la enseñanza globalizada y la correlación entre las asignaturas, siendo mencionados los métodos Montessori, Decroly, de proyectos y el plan Dalton; en el abordaje de la didáctica especial, destinada a la enseñanza de cada uno de los contenidos de la escuela primaria, estaban previstas la producción y el análisis crítico de materiales, la organización de excursiones, el estudio de los casos problemas, aspectos referentes a la evaluación y a la aplicabilidad de principios generales a los varios grados de enseñanza.

22 Op. cit., artículo 768.

23 Universidade de São Paulo. Instituto de EducaÇão: Programmas dos cursos regulares e extraordinários para o ano de 1936, São Paulo, Imprensa Oficial do Estado, 1936. 
Los objetivos generales de la renovación, expresados en la legislación, se materializaban en los programas a través de la lista de temas a ser desarrollados que, a su vez, podían ser identificados en los títulos de los libros o de los capítulos presentes en la bibliografía indicada para diferentes asignaturas. Tales indicaciones referenciaban un conjunto de autores (John Dewey, William Kilpatrick, Paul Monroe, A. M. Aguayo, E. L Thorndike, Lourenço Filho y Anísio Teixeira, por ejemplo) como portavoces de las nuevas tendencias educacionales, así como las iniciativas editoriales nacionales (Biblioteca de Educación, dirigida por Lourenço Filho y la Colección Actualidades Pedagógicas, dirigida por Fernando Azevedo) que las hacían asequibles a los lectores.

En el mismo año fueron publicadas las Sugestões de programas para as Escolas Normais $^{24}$ que, propuestas para que fuesen desarrolladas en instituciones con menos recursos administrativos que el Instituto de Educación, deberían acompañar la tendencia renovadora de él emanada, sin embargo, adaptadas a las condiciones existentes.

Elaboradas por los mismos profesores del Instituto de Educación, a los programas para las Escuelas Normales fueron añadidas explicaciones generales sobre los objetivos de cada asignatura y, algunas de ellas, tuvieron sus contenidos condensados en carga horaria menor, otras presentaban variaciones de la nomenclatura y de los objetivos. Un ejemplo de la subordinación al Instituto de Educación se encuentra en el programa de Psicología que, además de las cuestiones conceptuales, lista entre las actividades formativas «pesquisas referentes al aprendizaje» y la necesidad de que «cada tópico del programa será seguido de práctica en la escuela primaria adjunta»; además de eso sugiere «que en las vacaciones haya reuniones de los profesores de psicología en São Paulo, para elección y combinación de trabajos que puedan ser hechos generalmente en todos los cursos de formación de profesores ${ }^{25}$. El programa de Sociología de la Educación ejemplifica la necesidad de adaptación al presentar una lista más pródiga en sugestiones de investigaciones sociales sobre el medio, ciertamente más diversificado de lo que está aquel del Instituto de Educación.

Diferencias más significativas entre los dos tipos de instituciones pueden ser percibidas en la comparación de los programas directamente destinados a la formación profesional del profesor. En el Instituto de Educación, el programa de Asignaturas y Prácticas de la Enseñanza Primaria abarcaba los principios de la didáctica, los procesos metodológicos, los sistemas de enseñanza (Decroly, Montessori, de proyectos, entre otros) y la didáctica especial que consistía en la aplicabilidad de este conocimiento a los contenidos de la escuela primaria (lectura, escritura, lenguaje, cálculo, geografía, historia natural, higiene, dibujo, música, moral y educación cívica). En las Escuelas Normales, el programa de Práctica de Enseñanza incorporaba cuestiones sobre la organización escolar para que los futuros profesores

24 São Paulo. Secretaria da Educação e da Saúde Pública. Directoria de Ensino: «Sugestões para os Programmas das Escolas Normais», Boletim n. 2, São Paulo, 1936. Las escuelas normales, a difencia del Instituto de Educación, no ofrecían cursos de perfeccionamiento o de especialización.

${ }_{25}$ Op. cit., p. 7. 
«pudiesen sentir la situación de las soluciones aprendidas dentro de los problemas reales [...] de la escuela como es actualmente ${ }^{26}$ en las diferentes ciudades en detrimento de la didáctica específica de cada uno de los contenidos. No obstante, Música y Dibujo fueron también asignaturas del currículum del Curso Normal.

En 1938, el Directorio General de Enseñanza publicó un nuevo volumen dedicado a los programas de las Escuelas Normales ${ }^{27}$ tras el retorno del Instituto de Educación Caetano de Campos al nivel secundario de instrucción. En él, se enfatizada la importancia de las Escuelas Normales para el desarrollo de las ciudades del interior del estado. Según el documento, «de ellas vienen las prácticas y las ideas nuevas que poco a poco se infiltrarán» en todo el sistema; «en ellas se hacen las pesquisas, se apuran las observaciones, se vivifica la experiencia generadora de nuevas directrices ${ }^{28}$ y "por las conferencias de sus profesores, por sus asociaciones, por su biblioteca abierta a todos $»^{29}$ irradiarán nuevos estándares culturales.

El programa de la asignatura Práctica de Enseñanza fue reconfigurado y adquirió mayor detallamiento, estableciéndose que la formación profesional debería obedecer una secuencia de tres fases: observación, participación en los trabajos escolares y gobierno autónomo de la clase. Empezando por las cuestiones referentes a la decoración del aula y organización de los alumnos y del horario, abordaba la aplicación de tests en la evaluación y la adaptación del programa oficial al entorno donde será desarrollado. La participación de los estudiantes en las actividades de la escuela primaria progresaba con la preparación del material para la enseñanza, organización de excursiones, museos escolares y biblioteca primaria, alfabetización, enseñanza de la lectura, lenguaje oral y escrito, la adaptación del horario y del programa al sistema de proyectos.

$\mathrm{El}$ análisis de los programas revela la convergencia de las diferentes asignaturas para las pesquisas sobre el ambiente social. La Biología Educacional, por ejemplo, además de tratar de cuestiones relacionadas con la higiene y a la salud, debería dedicar especial atención a los estudios del medio, con la orientación para que «las clases de exposición del profesor sean alternadas con la presentación, por los alumnos, de los resultados, de sus lecturas y observaciones referentes a los asuntos del programa», sugiriendo «investigación respecto de las causas de ausencia de los alumnos de una escuela» y de sus hábitos higiénicos ${ }^{30}$. En la misma dirección, el contenido de la Sociología Educacional, que constituía la base científica de la formación junto con la Biología y la Psicología, debería enfatizar los trabajos prácticos, enseñando «a ver, observar y investigar y a hacer incidir esa observación sobre los hechos del entorno, que son más fáciles con observación directa» ${ }^{3 \mathrm{~T}}$.

26 Op. cit., p. I2.

27 São Paulo. Secretaria da Educação e da Saúde Pública. Directoria do Ensino: Boletim n. I7, Programma das Escolas Normais, 1938.

\footnotetext{
${ }_{28}$ Op. cit., p. 4.

Op. cit., p. 5 .

Op. cit., p. 25 .

Op. cit., p. 26.
} 
Así, los programas publicados en 1938 indican puntos de inflexión en los procesos de renovación de las prácticas y concepciones educacionales. Las Escuelas Normales ubicadas en el interior del estado, en 1933, obscurecidas por el Instituto de Educación Caetano de Campos, adquirieron una posición más relevante en el sistema y mayores atribuciones para el desarrollo de la práctica de enseñanza, núcleo de la formación profesional; además de eso, los programas de 1938 no están acompañados de bibliografía, indicativo probable de mayor autonomía conferida a los profesionales de esas instituciones.

La legislación y los programas aquí descritos, así como la concepción educativa que pretendían traducir, adquirieron estabilidad en el estado de São Paulo, conducida por la disminución de las discusiones y por el control de la esfera cultural en los años en que Getúlio Vargas ocupó la presidencia de la República (1937-1945) y solo fueron objeto de nuevas prescripciones en 1953. Ese periodo que quince años de estabilidad normativa es propicio al estudio de la dinámica de la circulación de las ideas de la Escuela Nueva en otro nivel del sistema educativo, cual sea, aquel responsable por la fiscalización de las normas legales y de la implementación programática.

\section{Modos de practicar}

En la estructura administrativa establecida por el Código de Educación de I933, a los delegados e inspectores de Enseñanza incumbía la fiscalización del cumplimiento de las normas legales, así como la orientación pedagógica para que ellas fueran llevadas a cabo. Las actividades educacionales cotidianas desarrolladas por profesores y alumnos, base de la estructura jerárquica del sistema, tenían como representantes formales los directores escolares que se reportaban a la fiscalización y a la orientación de los inspectores escolares, que, a su vez, daban cuenta al delegado de enseñanza que, sintetizando las informaciones y análisis de su región administrativa, contribuía al mapeo de la educación en el estado de São Paulo.

Los Informes ${ }^{32}$ elaborados por los delegados de enseñanza aquí analizados obedecían a un plan establecido por el director general de la Instrucción que incidía más detenidamente sobre los datos para la producción de la estadística escolar (descripción de edificios y condiciones materiales de las escuelas, matrículas, frecuencia de alumnos y profesores, horarios, festividades, estandarización de procedimientos) y, bajo la denominación Parte Pedagógica, eran presentadas sugerencias, reflexiones sobre el currículo y sobre métodos

32 El Archivo Público del estado de São Paulo tiene disponibles para consulta digital ejemplares, sin series completas, de informes de la educación producidos entre los años de I852 y 1945 (http://www.arquivoestado.sp.gov.br/site/acervo/repositorio_digital/relatorios_educacao). Entre los ejemplares disponibles, fueron seleccionados para este análisis los Informes de los delegados de Enseñanza de las regiones más prósperas del estado de São Paulo, o sea, de las ciudades de Campinas, São Carlos, Araraquara, Ribeirão Preto y São Paulo, la capital del estado, producidos entre los años de 1933 y 1945 . 
LA CIRCULACIÓN DE LA ESCUELA NUEVA EN BRASIL (I933-I958):

ENTRE PRESCRIPCIONES E INNOVACIONES

VERA TERESA VALDEMARIN Y MANUELA PRISCILA DE LIMA BUENO

de enseñanza, apreciación de los resultados obtenidos y reivindicaciones. A través de esas fuentes, se puede comprender cuáles aspectos de la renovación adquirieron lastre en el día a día de las escuelas; éstas revelan también la tensión instaurada entre la necesidad de cumplimiento de las normas legales y las condiciones existentes para su implementación.

Las discusiones pedagógicas, presentes en menor escala en los informes, indican que el nuevo léxico educacional fue incorporado al discurso de los delegados e inspectores de enseñanza. Autores, temas y prácticas identificados con las nuevas concepciones están presentes en las orientaciones para la enseñanza primaria. El sistema de proyectos y la enseñanza globalizada de los contenidos son recomendados como método para preparar a los alumnos para la vida práctica. Aunque la transición de la «escuela tradicional» o «de mera instrucción» para dicha «escuela nueva» o «escuela activa» sea lenta, deberían ser respetadas las bases previstas por Ferrièrre, en lo que se refiere a la adaptación a las leyes naturales del niño, y por Kilpatrick, en lo que se refiere a los cambios de la sociedad ${ }^{33}$.

En las orientaciones a los profesores, se estimulaba la disminución de las clases abstractas y el incremento de cuestiones relacionadas con la vida y con el entorno social ${ }^{34}$ y en la misma dirección, el Código de Educación era utilizado para justificar la necesidad de actividades basadas en la observación y en la experiencia personal del alumno, abriéndose, por esa vía, numerosas posibilidades pedagógicas con el uso de juegos educativos y excursiones, para hacer la escuela más interesante para el niño ${ }^{35}$.

John Dewey es el autor citado para justificar la realización de excursiones y de clases objetivadas que buscan «reconstruir y reordenar la experiencia de modo a habilitar el educando a conducirse, por uno mismo, el curso de sus futuras experiencias [...] una vez que el niño busca en la escuela el material para vivir, no entre las paredes de la educación, sino en el espacio libre, a lo largo del taller de la vida ${ }^{36}$. Sin embargo, además de los esfuerzos para la diseminación de nuevos métodos, se alega que la falta de cultura de los profesores formados por las Escuelas Normales constituye un impedimento y que «la renovación didáctica tendrá que ser ensayada primero en los centros más civilizados, viniendo después, hasta nosotros» ${ }^{37}$. Situados jerárquicamente entre el comando y la ejecución de las nuevas propuestas, los delegados de enseñanza equilibraban expectativas y seleccionaban las sugerencias «más oportunas y practicables en el perfeccionamiento y eficacia de los trabajos ${ }^{38}$, conforme se puede observar en el enfrentamiento de algunas cuestiones.

La autonomía didáctica, propuesta por el Directorio de Enseñanza para adaptar la educación al entorno en que era desarrollada, fue objeto de controversias

33 São Paulo: Relatório da Delegacia Regional de Ensino de Ribeirão Preto, 1936, p. II.

34 São Paulo: Relatório da Delegacia Regional de Ensino de São Carlos, I945.

35 São Paulo: Relatório da Delegacia Regional de Ensino da Capital, I943.

36 São Paulo: Relatório da Delagacia Regional de Ensino de São Carlos, 1945, p. 7.

37 SÃo Paulo: Relatório da Delegacia Regional de Ensino de Araraquara, 1936, p. II.

38 São Paulo: Relatório da Delegacia Regional de Ensino de São Carlos, 1933, p. I. 
persistentes. Podría ser adoptada condicionada a la existencia de una sólida cultura profesional de los profesore ${ }^{39}$ o con aplicación restricta, pues su uso indebido podría complicar el funcionamiento de la organización escolar ${ }^{40}$, además, para su concretización, sería necesaria la creación de un organismo orientador pues, aunque esté garantizada en el Código de Educación, no debe considerarse como regla absoluta: «Está limitada al campo de la pedagogía contemporánea y requiere autorización del Departamento de Educación» ${ }^{4}$. La excepción parece haber ocurrido en la ciudad de São Carlos, en 1939, donde el delegado afirma que la autonomía didáctica concedida «dentro de las aconsejadas por la pedagogía contemporánea» hizo de cada profesor «un colaborador consciente de las autoridades, en la realización de los trabajos educacionales, provocadores del mejor rendimiento escolar» ${ }^{42}$.

En su mayoría, los delegados de enseñanza parecen considerar la autonomía didáctica un complemento y no la antítesis de los programas prescritos, lo que, por cierto, facilitaría el trabajo de fiscalización por ellos realizado. Las reuniones pedagógicas se convirtieron en el espacio privilegiado para la adaptación de la enseñanza a las diferentes regiones del estado, en el cual los profesores presentaban «estudios de problemas pedagógicos de inmediata aplicación siempre con el objetivo de renovar las técnicas de enseñanza y corregir métodos fallos»43, o formaban equipos para estudio, discusión y de compartir experiencias ${ }^{44}$. Servían también para la mejora técnica y cultural de los profesores, una vez que eran ofrecidas palestras, clases, demostraciones y conferencias ${ }^{45}$. En la capital, en 1943, fue creada la sala de documentación didáctica, destinada al registro y a la permuta de materiales como, por ejemplo, planos globalizados ordenados por centros de interés o por proyectos ${ }^{46}$. Otra medida adoptada fue la creación del Centro de Estudios Pedagógicos, en la ciudad de São Carlos, en la cual «se puso de acuerdo, que las buenas ideas, aceptables, allí emitidas, fueran puestas en práctica, oyendo siempre a los directores, inspectores y la Delegación de Enseñanza» ${ }^{47}$.

En medio de las tentativas para adoptar las directrices renovadas y el enfrentamiento de las dificultades que se presentaban en las escuelas, se buscó un término medio. Una de las soluciones encontradas fue establecer que «habrá en los lunes, miércoles y viernes una globalización parcial de cuatro asignaturas: lectura, lenguaje oral, lenguaje escrito y dibujo. En los otros días martes, jueves y sábados, las clases serán impartidas como siguen siendo hasta ahora» ${ }^{4}$. Aunque parciales, esas iniciativas podrían ser permutadas entre los profesores de la red escolar, creándose,

39 São Paulo: Relatório da Delegacia Regional de Ensino de São Carlos, 1933.

40 São PaUlo: Relatório da Delegacia Regional de Ensino de Araraquara, 1936.

${ }_{41}$ São Paulo: Relatório da Delegacia Regional de Ensino da Capital, 1943, p. 72.

${ }^{42}$ São Paulo: Relatório da Delegacia Regional de Ensino de São Carlos, 1939, p. 2.

43 São Paulo: Relatório da Delegacia Regional de Ensino de São Carlos, 1942, p. 45.

${ }^{44}$ São Paulo: Relatório da Delegacia Regional de Ensino de Campinas, I94I.

45 SÃo Paulo: Relatório da Delegacia Regional de Ensino da Capital, I943.

46 Idem, ibidem.

47 S̃̃o Paulo: Relatório da Delegacia Regional de Ensino de São Carlos, 1945, p. 6.

${ }_{48}$ SÃo Paulo: Relatório da Delegacia Regional de Ensino da Capital, 1943, p. 35. 
LA CIRCULACIÓN DE LA ESCUELA NUEVA EN BRASIL (I933-I958):

ENTRE PRESCRIPCIONES E INNOVACIONES

VERA TERESA VALDEMARIN Y MANUELA PRISCILA DE LIMA BUENO

así, un «repositorio de documentación de los trabajos», que no deben «ser simplemente copiados, porque sería salir de una rutina para incidir en otra aun peor, ahora entonces encubierta con las lentejuelas de una pretendida técnica mejor» ${ }^{49}$.

El manejo de los procedimientos de investigación también parece tener establecidas continuidades prácticas en el sistema, pues, además de la recomendación de la constitución de un repertorio de experiencias, podría ser utilizado para elevar el número de niños en la escuela, investigándose las causas de la evasión. El delegado de enseñanza de la ciudad de São Carlos creó un instrumento que, apoyado en las técnicas de estadísticas, posibilitó evaluar la eficiencia de la enseñanza en su región: elaboró un test, que fue aplicado a los alumnos de la escuela, cuyos resultados demostrarán que la enseñanza era muy verbalista y que las asignaturas más propicias a la resolución de los problemas deberían ser enfatizadas en la orientación a los profesores, lo que demandaría la inversión en estudios de casos e investigación en la biblioteca ${ }^{50}$. Hubo también sugerencias para que en las escuelas de la capital del estado fueran realizadas investigaciones teniendo como foco la salud de los alumnos, la falta de éxito en el aprendizaje y la organización de las clases por criterios de rendimiento ${ }^{\text {II }}$.

A pesar de las iniciativas descritas, sobresalen en esas fuentes los relatos de las dificultades encontradas para que la renovación se efectuara. La principal barrera apuntada por los delegados sería proveniente de deficiencias relativas a la formación de los profesores (que presentaban graves fallas en lo que se refiere a la práctica de enseñanza) y a la selección de equipo docente. Las normas legales, que encargaban a los inspectores diseminar los nuevos procesos de enseñanza entre profesores y directores, parecen haber sido anuladas por el exceso de trabajo administrativo, que consumía tiempo de la orientación pedagógica. Las largas distancias a recorrer entre las ciudades eran agravadas por la demora en el recibimiento de asignaciones necesarias para los viajes ${ }^{52}$ y por la gran cantidad de escuelas a ser orientadas ${ }^{33}$. Incluso reconociendo las cualidades de la legislación, el exceso de centralización en las decisiones educacionales demandaba mayor número de inspectores que pudiesen efectivamente enseñar haciendo uso de su propia experiencia y de la bibliografía disponible ${ }^{54}$.

49 Idem, p. 8I.

so São Paulo: Relatório da Delegacia Regional de Ensino de São Carlos, 1940.

sı São Paulo: Relatório da Delegacia Regional de Ensino da Capital, I943.

52 La misma justificación se encuentra en diversos informes: São PaUlo: Relatório da Delegacia Regional de Ensino de São Carlos, 1933 y São Paulo: Relatório da Delegacia Regional de Ensino de Ribeirão Preto, 1936.

53 São Paulo: Relatório da Delegacia Regional de Ensino de Araraquara, 1940; São Paulo: Relatório da Delegacia Regional de Ensino de São Carlos, 1945; SÃo Paulo: Relatório da Delegacia Regional de Ensino da Capital, I943.

${ }_{54}$ Es grande el número de inspectores escolares que publicaron manuales didácticos para el uso de profesores en formación o en ejercicio en el periodo, en los cuales, posiblemente, sintetizaban las orientaciones dadas en las escuelas, valiéndose de la estrategia de diseminar nuevas prácticas a través de publicaciones especializadas. Ver, al respecto, Valdemarin, V. T. y Campos, D. G. S.: «Concepções pedagógicas e método de ensino: O manual didático Processologia na Escola Primária», Paideia, vol. 17, n. 38 (set-dez 2007), pp. 343-356 y Valdemarin: 20Io, op. cit. 
La falta de atractivos de la carrera docente -ingreso en condiciones precarias, bajos sueldos, falta de estabilidad-, alineada a la precariedad material de muchas escuelas ${ }^{55}$, llevan a la evaluación de que la legislación era difícil de ser cumplida ${ }^{56}$ y a la propuesta de la creación de clases experimentales para el ensayo de nuevas prácticas, al ofrecimiento de cursos y a la publicación de periódicos ${ }^{57}$. Al gran número de problemas enfrentados se sigue la constatación de que el estado de São Paulo perdió el liderazgo en la educación porque la legislación desconoció las posibilidades y los límites de cada municipio ${ }^{58}$ y sigue viviendo de antiguos ideales.

Las fuentes aquí analizadas indican la complejidad constitutiva de los procesos de cambio cultural y pedagógico. Es innegable que las directrices renovadas fueron incorporadas al léxico de los profesionales de la educación, que pasaron a utilizar las nuevas expresiones para reivindicar medidas y recursos, para describir innovaciones o tentativas y para adherirse al discurso oficial. Esa forma discursiva contribuyó a caracterizar iniciativas que, aunque específicas, confirmaban las potencialidades de los propósitos pretendidos. Sin embargo, el volumen de las dificultades que se presentaban, entre las dimensiones del sistema y los mecanismos del control, evidenciaban la apropiación selectiva inherente a los procesos de innovación de las prácticas pedagógicas, de instauración más lenta o más dependiente de los recursos coyunturales que las prácticas discursivas, a ser directamente experimentados por actores e instituciones. Ese desequilibrio parece actuar como fomento para las propuestas legales subsecuentes.

\section{La renovación apropiada}

Al fin del gobierno dictatorial de Getúlio Vargas, en 1946, una nueva Constitución entró en vigor en el país y de ella partieron cambios substanciales en todos los sectores. Para los propósitos de este artículo, importa destacar la promulgación de la Ley Orgánica de la Enseñanza Normal'9 que establecía las directrices comunes nacionales para la formación de profesores, a las cuales los estados brasileños deberían adaptarse. En el estado de São Paulo, sin embargo, no hubo iniciativas inmediatas para el cumplimiento de esa determinación: solamente en 1950 fue instituido el Reglamento Interno de las Escuelas Normales ${ }^{60}$ y solamente en 1953 fueron publicados nuevos programas para el Curso Normal ${ }^{61}$,

"São Paulo: Relatório da Delegacia Regional de Ensino de São Carlos, 1940.

56 São Paulo: Relatório da Delegacia Regional de Ensino de Araraquara, I940.

57 São Paulo: Relatório da Delegacia Regional de Ensino da Capital, 1943.

58 São Paulo: Relatório da Delegacia Regional de Ensino de São Carlos, 1943.

59 BRAsil: «Decreto-Lei n. 8.530, de 2 de enero de 1946. Lei Orgânica do Ensino Normal», Diário Oficial da República Federativa do Brasil, Rio de Janeiro, 1946.

60 SÃo Paulo: Decreto n. 19.525-A, de 27 de junho de 1950. Regimento Interno das Escolas Normais.

${ }_{61}$ São Paulo. Secretaria do Estado dos Negócios da Educação. Serviço de LegislaÇão e Publicidade: «Programas das Escolas Normais do estado de São Paulo», en Compêndio de Legislação do Ensino Normal, São Paulo, J. Bignardi \& Cia., n. 4, mar. 1953. 
a ser desarrollados en carácter experimental. Dicha demora no implicó el abandono de las concepciones escolanovistas, sino su reiteración.

Esa normalización presenta una alteración curricular importante: el primer año del Curso Normal pasó a ser denominado Curso Pre-Normal y en él fueron reunidas asignaturas destinadas a promover un repaso de los conocimientos generales (Lengua Portuguesa, Matemáticas, Ciencias Físicas y Biológicas, Historia, Dibujo y Música). Bajo la denominación Curso Normal fue organizado el conjunto de asignaturas exclusivamente dedicado a la formación profesional. Esa opción indica el intento de mejorar la calidad en la formación de profesores en dos direcciones: la primera de ellas, reforzando el contenido propedéutico con la creación de un curso de transición -o Curso Pre-Normal- y a la segunda, enfatizando la aplicabilidad del contenido educacional.

Los temas que componen los programas de las asignaturas reproducen, con pequeñas alteraciones, aquellos publicados en 1938 y, en la introducción de cada uno de ellos, se presentan sus finalidades para la «formación de técnicos para el magisterio primario» ${ }^{62}$, con la recomendación de que «las clases deben ser siempre acompañadas de trabajos prácticos» ${ }^{63}$. Esos programas organizan los conocimientos para que sean cumplidas las directrices didácticas expresados en el Reglamento de las Escuelas Normales, como son «los conocimientos necesarios a la comprensión y solución de los problemas concretos de educación y enseñanza, a la medida que van surgiendo en el contacto diario de los normalistas con las clases del curso primario» ${ }^{64}$.

El programa de Práctica de Enseñanza, núcleo de esa estructura, que se desarrollaría a través de la creación de oportunidades para el ejercicio de la observación, coparticipación y de la regencia de clase, reitera la articulación estrecha entre Curso Normal y Escuela Primaria y especifica el uso de excursiones, de proyectos y de la estadística como recursos a ser trabajados en la formación del profesor. El programa de Biología, además de incluir cuestiones referentes a la salud en el entorno rural, prescribe que «los alumnos harán estudios estadísticos y gráficos sobre nupcialidad, la natalidad, la mortalidad general y la mortalidad infantil del municipio» ${ }^{65}$; el programa de Sociología indica que, en esa área, «como de cualquier otra ciencia, enseñar antes de todo a ver, observar y investigar y a hacer incidir esa investigación sobre hechos del entorno local, que son más fáciles de observación directa» ${ }^{66}$, además de sugerir temas para pesquisas.

Un año después, en 1954, nuevos Programas de las Escuelas Normales ${ }^{67}$ fueron publicados afirmándose en la Introducción que «siguen válidas en la homogeneidad de su conjunto las claras y siempre oportunas recomendaciones que acompañarán

62 Op. cit., p. I5.

63 Op. cit., p. $3 \mathrm{I}$.

${ }^{64}$ SÃo Paulo. Decreto n. 19.525-A, de 27 de junio de 1950. Regimento Interno das Escolas Normais, Artigo 70.

6s Op. cit., p. 27.

66 Op. cit., p. 3I.

${ }_{67}$ São Paulo: Portaria n. 49, do Departamento de Educação, de 04 de diciembre de 1954. 
a los añejos programas» $y$, para reforzar estas intenciones, fue transcrita íntegramente la justificación que acompañaba a los programas de 1938.

Además de esa declaración de principios, la recogida entre los dos documentos evidencia que se trataba de nuevos programas. En primer lugar, cambiaron los proponentes retirándose el equipo docente del Instituto de Educación Caetano de Campos para dar espacio a la colaboración de profesores de la Facultad de Filosofía y de comisiones técnicas. Otros cambios provienen de la ampliación del número de instituciones formadoras; los programas de 1938 se destinaban a las nueve Escuelas Normales que funcionaban en el estado y los nuevos deberían ser aplicados «sin contar los institutos de educación de creación más reciente, en las ochenta y tres escuelas oficiales, dieciséis municipales y ciento treinta y dos libres, que funcionan en nuestro estado», dificultando el control sobre su cumplimiento.

Los análisis de los Programas de 1954 revelan que el Curso Pre-Normal fue mantenido, pero hubo inflexión en los temas y en los contenidos. Los temas del programa de la asignatura de Pedagogía se volvieron menos específicos en esa versión, siendo mencionados, por ejemplo, «visión general sobre la escuela nueva», «estudios sobre los métodos generales y algunos métodos de enseñanza» y «renovación escolar en función de la realidad nacional». El programa de Sociología de la Educación, además de contribuir a la adquisición de cultura general, debería posibilitar la comprensión de la función social y de las limitaciones de la escuela en el conjunto de las instituciones sociales y, además de la supresión de las sugerencias de temas para investigación, se recomienda al profesor que explicite «relaciones y sistematizaciones, a partir de su adecuación terminológica, hasta llegar a la máxima teorización que la edad de los alumnos y el conjunto de sus estudios lo permitan» ${ }^{68}$.

El Programa de Práctica de Enseñanza, que reunía elementos teóricos y oportunidad para la "práctica efectiva del magisterio», previo estudio de programas enseñados en la escuela primaria y la realización de «pasantías direccionadas en las clases de aplicación y asistencia de clases-modelos impartidas por el profesor de Práctica», además de la coparticipación en las actividades y elaboración de planes de curso y de clases. Los temas abarcan la legislación del magisterio, organización de la clase y del horario, visitas de estudios y «práctica de los métodos modernos de estudio».

De esta forma, además de la declaración inicial sobre el mantenimiento del mismo espíritu animando las ediciones de 1938 y de 1954 de los programas para las Escuelas Normales, la concepción de la Escuela Nueva se ha reducido en el léxico y en las prácticas a ella asociadas, como, por ejemplo, la investigación como actividad formativa y las indicaciones bibliográficas como base de lecturas y propuestas.

El tiempo de estabilidad en la legislación estaba acabado y, con él, la concepción que animó las reformas de la década de 1930. La justificación que seguía la propuesta de nuevas alteraciones, en 1957, aclaraba ese propósito:

68 Idem. 
LA CIRCULACIÓN DE LA ESCUELA NUEVA EN BRASIL (I933-I958): ENTRE PRESCRIPCIONES E INNOVACIONES

VERA TERESA VALDEMARIN Y MANUELA PRISCILA DE LIMA BUENO

La estructura dada en 1933 por el Código de Educación a la enseñanza normal en São Paulo desfigurada por la legislación posterior, superada también que fue por las circunstancias sociales y pedagógicas supervinientes, ya no sirve más, como tan bien servía anteriormente, a las necesidades educacionales para cuya satisfacción existe $^{69}$.

Además del epitafio de la concepción, se citan los problemas más urgentes a los cuales se enfrentaban las autoridades educativas -ingreso facilitado en los cursos de formación de profesores, ausencia de criterios para aprobación en las asignaturas, existencia de escuelas diferentes para la misma certificación- $y$, para solucionarlos, fueron propuestos la extinción del Curso Pré-Normal, la creación de exámenes para ingreso, la unificación de los currículos con la adopción de aquel ya existente en los Institutos de Educación, la inclusión de las asignaturas de Educación Social y Cívica y Lengua Portuguesa en los currículos, el establecimiento de nota mínima para aprobar en todas las asignaturas y la creación de cursos de Perfeccionamiento en las Escuelas Normales, antes prerrogativa de los Institutos de Educación. Y, para no dejar ninguna duda sobre los cambios de orientación, se afirma el deseo de «huir a las reflexiones de orden académico o de sentido elegantemente teórico, sin perder por un solo instante la realidad de São Paulo» ${ }^{\circ}$.

A causa de ese análisis fue establecida una nueva organización para la Enseñanza Normal $^{71}$ y en 1959 fueron publicados nuevos programas ${ }^{72}$. Elaborados por diversas comisiones compuestas por profesores y técnicos en educación, evidencian la aglutinación de temas, sin indicación bibliográfica o autores referenciales. Constituyen, por lo tanto, un documento propicio para indicar los aspectos de la renovación desencadenada en los años 1930 que se arraigaron en las normalizaciones.

Para la asignatura denominada Pedagogía y Psicología General y Educacional se indicaba el estudio de los «aspectos positivos de los diversos sistemas de educación y posibilidad de aprovechamiento en la escuela actual» ${ }^{73}$, pero la prioridad era concedida a las cuestiones sobre el desarrollo y aprendizaje; la asignatura de Metodología y Práctica de la Enseñanza Primaria, con carga horaria grande y presente en las tres series del curso, reunía orientación para la observación en las clases del curso primario, el estudio de sus programas y, al final, orientaba a la «realización práctica de centros de interés, proyectos y unidades de trabajo», el desarrollo de los métodos de educación renovada y las «clases globalizadas, unidades de trabajo, incluyendo la práctica de regencia de cantos cívicos y canciones y la ilustración didáctica, de conformidad con los programas de Música y Canto Orfeónico y Dibujo Pedagógico, respectivamente» ${ }^{74}$.

69 SÃo Paulo. Secretaria de Estado dos Negócios da Educação. Legislação do Ensino Normal no estado de São Paulo actualizada hasta 30 de abril de 1960, p. 9.

70 Op. cit., p. Iо

${ }_{71}$ Lei n. 3.739, de 22 de enero de 1957. Dispone sobre la organización do enseñanza normal en el estado de São Paulo. Decreto n. 35.I0o, de I7 de junio de 1959.

72 Op. cit., p. 40.

73 Op. cit., p. 40.

74 Op. cit., p. 42. 
En ese recorrido, se puede constatar la pérdida de la concepción escolanovista, sin que otra haya, precisamente, tomado su lugar.

\section{La circulación de ideas pedagógicas}

Las fuentes documentales aquí analizadas posibilitan comprender el entrelazamiento de las acciones desencadenadas con el objetivo de renovar las prácticas pedagógicas en Brasil, en la primera mitad del siglo xx.

En ese proceso, las concepciones tributarias de la Escuela Nueva o Educación Progresiva constituyeron la base para la introducción de cambios significativos en el sistema educacional brasileño y, de modo particular, en el estado de São Paulo.

Uno de los sentidos más denotativos de las innovaciones pretendidas fue la remodelación de la organización administrativa, entendida como estructura capaz de poner en movimiento las nuevas prácticas pedagógicas o, de otra manera, alineando los modos de pensar a los modos de hacer.

Bajo el liderazgo de un grupo de intelectuales que ocupaba puestos clave en la jerarquía política, académica y editorial, la legislación desempeñó un papel fundamental en la estrategia de circulación de nuevas ideas, sirviendo tanto para una red articulada de avance como para prescribir el guion de lecturas y de prácticas deseadas. A la legislación estaban articuladas iniciativas editoriales que irradiaban diferentes interpretaciones destinadas a cambiar la mentalidad de los profesores. Con estos medios a disposición fue posible hacer de la Escuela Nueva una concepción hegemónica que se presentaba como ruptura y como innovación y se expresaba a través de un glosario específico, estableciendo áncoras a través del lenguaje.

En esa estrategia, diferentes instituciones fueron identificadas como sitios para la irradiación de ideas y prácticas. En la primera etapa, el Instituto de Educación Caetano de Campos, ocupando la posición modelo y funcionando como caja de resonancia para incitar las posibilidades inherentes a la renovación. En una segunda etapa, la diseminación de las escuelas normales y la consecuente ampliación de plazas para la formación de profesores. En esa expansión, sin embargo, las pretensiones de la reforma empiezan a evidenciar límites y contradicciones, entre ellas, las dificultades para equilibrar las determinaciones legales con las demandas de los diversos contextos, la expectativa depositada en la formación de profesores y la diseminación de prácticas en un sistema educacional que adquirió grandes proporciones.

Es innegable que tal expansión contribuyó a la creación de un repositorio de nuevas prácticas que, aunque en pequeña proporción, se arraigaban en procedimientos metodológicos que permanecieron en la legislación, perdieron la connotación de innovación, por ejemplo, las clases globalizadas, los centros de interés y la enseñanza por unidades o proyectos. Otras prácticas, tales como la pesquisa y la investigación, tuvieron vida cíclica y sucumbieron, probablemente, debido a su incompatibilidad con el proceso de prescripción. 
LA CIRCULACIÓN DE LA ESCUELA NUEVA EN BRASIL (I933-I958):

ENTRE PRESCRIPCIONES E INNOVACIONES

VERA TERESA VALDEMARIN Y MANUELA PRISCILA DE LIMA BUENO

La temporalidad inherente a los procesos que se presentan como nuevos evidenció la tensión entre concepciones y experiencias prácticas. Los programas, forma prescriptiva utilizada para, simultáneamente, introducir la innovación y controlar el sistema, documentaron el ciclo en lo cual los elementos que se presentaban como nuevos fueron selectivamente incorporados al sistema y a una tradición que contribuye activamente a la determinación de los significados sedimentados. 